Meta

Journal des traducteurs

Translators' Journal

\title{
La raison sociale en droit québécois
}

\section{Antoni Dandonneau}

Volume 24, numéro 1, mars 1979

La traduction juridique

URI : https://id.erudit.org/iderudit/002454ar

DOI : https://doi.org/10.7202/002454ar

Aller au sommaire du numéro

Éditeur(s)

Les Presses de l'Université de Montréal

ISSN

0026-0452 (imprimé)

1492-1421 (numérique)

Découvrir la revue

Citer cet article

Dandonneau, A. (1979). La raison sociale en droit québécois. Meta, 24(1),

166-176. https://doi.org/10.7202/002454ar d'utilisation que vous pouvez consulter en ligne.

https://apropos.erudit.org/fr/usagers/politique-dutilisation/ 


\section{La raison sociale en droit québécois}

Au Québec, selon un usage relativement récent, on entend par raison sociale le nom sous lequel un groupement, quelle qu'en soit la forme, ou même un simple commerçant exerce son activité. C'est ainsi qu'il faut entendre l'article 63 de la Charte de la langue française (L.Q. 1977, au chap. 5) : «Les raisons sociales doivent être en langue française. » $\AA$ première vue, du fait que cette disposition est édictée sous le titre « La langue du commerce et des affaires $»$, on pourrait croire qu'elle ne vise que les entreprises commerciales, de l'entreprise individuelle ${ }^{1}$ au type de société que notre droit appelle compagnie. Cependant, les articles 70 et 71 , rangés, pour des raisons que l'on s'explique mal, sous le même titre, contiennent des dispositions visant la raison sociale des associations sans but lucratif, des services de santé et des services sociaux.

En donnant au terme raison sociale un sens aussi étendu, le législateur a pu s'appuyer sur les travaux de la Régie de la langue française. En effet, un ouvrage publié par cet organisme ${ }^{2}$ déclare : " Il s'agit ici des raisons sociales, au sens large du terme, c'est-à-dire du nom que se donne une entreprise, une association, un commerçant. » Toutefois, quelques pages plus loin ${ }^{3}$, le même ouvrage restreint l'emploi du terme raison sociale au seul domaine commercial et même, semble-t-il, à l'entreprise exploitée en société : "Toute entreprise voulant faire affaire au Québec doit se donner un nom qui lui confère une personnalité juridique distincte. Pour les besoins de notre étude, nous appellerons ce nom «raison sociale » quel que soit le type de constitution juridique auquel il se rapporte. » Les réserves de ces deux textes i $($ au sens large du terme », " pour les besoins de notre étude ») et leur incompatibilité apparente auraient dû inciter le législateur à la prudence.

On peut d'abord remarquer, au nom du simple bon sens, qu'il paraît paradoxal d'appliquer le terme «raison sociale » à des personnes physiques; en continuant dans cette voie, on en viendrait à parler du siège social du commerçant plutôt que de son établissement. La même observation s'impose dans le

1. Il y a lieu de faire remarquer que ce terme est l'équivalent exact des termes anglais sole proprietorship ou single proprietorship; on voit souvent dans nos textes un équivalent qui semble influencé par l'anglais, «entreprise à propriétaire unique ».

2. Régie de la langue française, Comment formuler une raison sociale. Règles d'écriture des raisons sociales, Québec, Editeur officiel, 1975, p. 4.

3. Ibid., p. 9. 
cas de personnes morales qui n'empruntent aucun des traits de la société, par exemple les services sociaux. D'autre part, rien ne permettant de poser à priori que l'usage et le bon sens font toujours bon ménage, on ne peut remettre en cause l'emploi de raison sociale sans prendre en compte la langue juridique des divers pays (entièrement ou partiellement...) de langue française. Certes, le juriste mettra en garde contre les confusions que peut entraîner l'assimilation hâtive d'institutions différentes, mais il ne pourra faire que la rigueur terminologique perde ses droits, d'autant qu'à ses heures il sait mettre en valeur le sens des mots.

Dans la présente étude, qui se situe à la rencontre de la terminologie et du droit comparé, il s'agit donc de résoudre un certain nombre de questions tournant autour du terme \& raison sociale s. Peut-on l'employer aussi bien à propos de personnes physiques que de groupements ? Convient-il à n'importe quel type de groupements, qu'ils soient constitués ou non en vue d'un but lucratif ? Est-on justifié de l'appliquer à toutes les formes de société ? Peut-on appeler "raison sociale » un nom quelconque, abstraction faite de toute règle de composition? Enfin, dans les cas où l'une de ces questions appellerait une réponse négative, quels termes devraient prendre la relève?

\section{La raison sociale et la dénomination sociale en droit français}

En matière de sociétés, le Code civil du Québec emprunte bon nombre de dispositions au Code civil et au Code de commerce de France, ce qui nous justifie de commencer par l'étude du droit français. Dans le Code de commerce du $\mathrm{XIX}^{\mathrm{e}}$ siècle, la raison sociale ${ }^{4}$ est la désignation d'une société par le nom de tous les associés ou de certains d'entre eux (par exemple, Dupont et Durand, Durand et Compagnie 5. D'autre part, ne peuvent figurer dans la raison sociale

4. On trouvait aussi le terme « nom social », employé dans le même sens aux articles 23 et 29 ; voir E. Thaller et P. Pic, Des sociétés commerciales, 3e éd. par J. Kréher, Paris, Rousseau, 1940, p. 395, note $4:$ : l'on s'accorde à admettre que les deux expressions, raison sociale et nom social, ont exactement la même signification. 》D'ailleurs, les articles 19 et 27 du Code de commerce belge, parallèles aux articles du Code français, emploient \& raison sociale \$ au lieu de \& nom social ». De cet usage ancien de nom social, on distinguera soigneusement l'usage de certains auteurs récents qui entendent par «nom social \$ le nom d'une société, quelle qu'en soit la forme, donc aussi bien la dénomination sociale que la raison sociale. On trouvera des exemples de cet emploi récent dans J. Hémard, F. Terré et $P$. Mabilat, Sociétés commerciales, t. I, Paris, Dalloz, 1972 , p. 231-232, no 248, et dans D. Bastian et P. Nocquet, \& Raison sociale et dénomination sociale 》, Juris-classeur des sociétés, fasc. 28,1972 , nos 1,10 et 12 .

5. L'expression « et Compagnie \$ (abrégée en « et Cle 》) correspond aux expressions anglaises and Company, \& Co. ou and Associates et n'a rien à voir avec ce que nous appelons au Québec « compagnie »; elle est réservée, en droit français, aux sociétés en nom collectif et aux sociétés en commandite, ce qui exclut qu'on puisse l'employer dans le nom d'une société anonyme, forme française qui correspond à la «compagnie » du Québec. Elle vise simplement à indiquer l'existence d'associés autres que ceux dont le nom apparaît dans la raison sociale. Notons en passant qu'on semble employer en Belgique l'abréviation \& \& Co $\gg$ (L. Frédéricq, Traité de droit commercial belge, t. IV, Gand, Feycher, 1950, p. 311 et 318). D'autre part, l'expression \& et Associés », que l'on emploie souvent au Québec, ne semble pas attestée en Europe. En plus de a et Compagnie », l'usage français connaît aussi \& et autres », dans le cas des sociétés civiles professionnelles; on trouvera les lois relatives à ces sociétés dans le Code des sociétés, Paris, Dalloz, 1977. 
que le nom des associés indéfiniment responsables; ainsi, le commanditaire n'étant tenu qu'à concurrence de son apport, son nom est exclu de la raison sociale. On peut donc poser deux règles concernant la raison sociale : 1) une règle de coimposition : elle est formée exclusivement du nom des associés ${ }^{6}$, et 2) une règle d'emploi : elle est réservée aux sociétés comprenant des associés indéfiniment responsables, c'est-à-dire surtout aux sociétés de personnes ?

Quant à la société anonyme, elle tire son nom du fait qu'elle n'est pas désignée par une raison sociale, mais par l'objet de son entreprise (art. 29 et 30 du Code de commerce). Toutefois, cette disposition n'a pas toujours été respectée, car bon nombre de sociétés anonymes se sont donné une dénomination qui, sans prendre la forme d'une raison sociale, ne renseignait aucunement sur leur objet (dénomination de fantaisie ou comprenant un nom propre). Lors de la réforme de 1966, le législateur n'a pas jugé bon de définir la dénomination sociale et s'est contenté de disposer que «la société par actions est désignée par une dénomination sociale ${ }^{8} \gg$. On pourrait donc formuler de la façon suivante la règle de composition de la dénomination sociale : par opposition à la raison sociale, elle ne peut être formée exclusivement du nom des associés. Cependant, on ne peut établir de règle d'emploi symétrique à celle qui se dégage pour la raison sociale, puisque, en dehors du domaine commercial, la société civile (dans laquelle les associés sont indéfiniment responsables) peut aussi être désignée par une dénomination sociale ${ }^{\text {. }}$.

Pour l'essentiel, la réforme de 1966 n'a fait que reprendre la distinction entre raison sociale et dénomination sociale, telle qu'elle avait été établie au $\mathrm{XIX}^{\mathrm{e}}$ siècle. Cependant, force nous est de constater que cette distinction n'a pas toujours été respectée, notamment dans la jurisprudence ${ }^{10}$. Chose plus grave, le législateur lui-même avait fait une entorse à cette distinction dans la loi relative aux sociétés à responsabilité limitée, dont l'article 11 avait la teneur suivante : «La société à responsabilité limitée est, soit qualifiée par la désignation de l'objet de son entreprise, soit désignée sous une raison sociale comprenant les noms d'un ou de plusieurs associés ${ }^{11}$. $\gg$ Cette anomalie, qui allait directement à l'encontre de la règle d'emploi définie plus haut (puisque cette société ne comprend aucun associé tenu indéfiniment du passif social), a été supprimée lors de la réforme de 1966 ; en effet, la nouvelle loi porte que cette société \& est désignée par une dénomination sociale, à laquelle peut être incorporé le nom

6. Cette exclusion s'étend non seulement aux noms de tiers, mais aussi aux indications qui sont étrangères au nom des associés (Etablissements, Maison, etc.); voir J. CalaisAuloy, dans Répertoire des sociétés, t. III, Paris, Dalloz, s.v. Raison sociale, $\mathrm{n}^{\circ} 5$.

7. Le cas particulier de la société en commandite par actions est laissé de côté, d'autant que le droit québécois n'a pas retenu cette forme de société. Le lecteur qui désire étudier la question se reportera aux ouvrages déjà cités, Bastian et Nocquet ou Hémard, Terré et Mabilat.

8. Loi du 24 juillet 1966 sur les sociétés commerciales, art. 70.

9. Par exemple, Décret du 1er juillet 1971 relatif a certaines sociétés civiles faisant publiquement appel d̀ l'épargne, art. 2, 3, 7, 8 et 9. Voir également Bastian et Nocquet, op. cit., $\mathrm{n}^{\mathrm{OB}} 126$ et 127 ; cependant ces auteurs ne font pas mention des sociétés civiles professionnelles, désignées par une raison sociale (Loi du 29 novembre 1966 relative aux societés civiles professionnelles, art. 8 ).

10. Voir les décisions citées par Bastian et Nocquet, op. cit., $\mathrm{n}^{\circ} 132$.

11. Loi du 7 mars 1925 sur les sociétés à responsabilité limitée. 
d'un ou de plusieurs associés ${ }^{12} \gg$. La distinction entre ces deux types de désignation est désormais uniformément appliquée dans les textes législatifs français et on la retrouve dans le Code de commerce de Belgique. Toutefois, on trouve des textes publiés par l'Administration française qui emploient raison sociale, soit au lieu de dénomination sociale, soit comme terme générique englobant à la fois raison sociale et dénomination sociale ${ }^{13}$.

Si la société anonyme, du fait qu'aucun des associés n'y est indéfiniment responsable, ne peut être désignée par une raison sociale, peut-on dire, à l'inverse, que la société de personnes ne peut être désignée par une dénomination sociale? Au xIXe siècle, il était admis qu'une société de personnes, tout comme un commerçant, exploite un établissement sous une raison de commerce ou raison commerciale ${ }^{14}$; par exemple, une société en nom collectif dont la raison sociale était Durand et Compagnie pouvait exploiter un établissement sous la raison commerciale "Maison de la belle fermière ». Cette raison commerciale se distinguait doublement de la raison sociale : d'une part, elle désignait un établissement et non une société, d'autre part, elle était librement formée, de la même façon que la dénomination sociale ${ }^{15}$. Cependant, pour être claire en théorie, la distinction entre l'établissement et son titulaire n'en est pas moins difficile à établir dans la pratique : les ressources limitées de la société de personnes font qu'elle n'exploite bien souvent qu'un seul établissement. Du reste, les termes " raison commerciale » et « raison de commerce» sont de moins en moins employés.

Lors de la réforme du droit des sociétés, le législateur a voulu reconnaître expressément le droit pour la société de personnes d'utiliser un nom différent de la raison sociale; ce droit est établi par la disposition suivante : « Un nom commercial, distinct de la raison sociale, peut être utilisé par la société ${ }^{16}$. \ Le terme «nom commercial » est généralement défini : l'appellation sous laquelle une personne fait le commerce ${ }^{17}$. Si ce terme a supplanté raison commerciale, il ne s'ensuit pas qu'il en soit un parfait synonyme. Abstraction faite de la distinction entre l'établissement et son titulaire, il faut voir que \& nom commer-

12. Loi du 24 juillet 1966, déjà citée, art. 34.

13. Premier cas : les Cahiers français, no 141,1970 , p. 15 et no 189, p. 26, où l'on donne sous \& raison sociale » le nom des plus grandes entreprises françaises; il va de soi que toutes ces entreprises sont constituées sous la forme de sociétés anonymes. Second cas : dans un bon de commande accompagnant la revue Marchés publics, une mention indique : \& Nom, Prénom, ou Raison sociale $>$; on ne peut en conclure que la revue compte exclure du nombre de ses abonnés les sociétés anonymes.

14. On trouve aussi dénomination commerciale.

15. Voir H. Allart, Traité théorique et pratique des marques de fabrique et de commerce, Paris, Rousseau, 1914, p. $34, n^{\circ} 34 ; \mathrm{C}$. Chevenard, Traité de la concurrence déloyale en matière industrielle et commerciale, Genève, Kundig, 1914, p. 68 ; Nouveau Répertoire de droit, $2 \mathrm{e}$ éd., Paris, Dalloz, 1964, t. III, s.v. nom commercial, ${ }^{\circ} 27$. Il semble inexact d'affirmer, comme le font Bastian et Nocquet, op. cit., no 14 : «Bien souvent, les mêmes termes serviront tous à la fois d'enseigne et de raison commerciale, et ils constitueront en outre la raison sociale... de la personne morale exploitant l'entreprise. \$

16. Décret du 23 mars 1967 sur les sociétés commerciales, art. 8, al. $1 \mathrm{er}$.

17. Hémard, Terré et Mabilat, op. cit., p. 232, $\mathrm{n}^{0} 248$; Bastian et Nocquet, op. cit., $\mathrm{n}^{\circ} 12$; la confusion possible avec le nom de l'établissement n'est pas entièrement dissipée, comme le montre cette autre définition : * la dénomination sous laquelle est connu et exploité un fonds de commerce » (J.-P. Sortais, dans Répertoire des sociétés, t. III, Paris, Dalloz, s.v. Société anonyme, no 38. 
cial » est un terme générique pouvant englober à la fois le nom patronymique du commerçant, la raison commerciale, la raison sociale et la dénomination sociale, d'où la nécessité pour le législateur de préciser qu'il est « distinct de la raison sociale ${ }^{18}$ ». Mais ce «nom commercial, distinct de la raison sociale» ne se confond-il pas avec la dénomination sociale ? Certains auteurs n'hésitent pas à l'affirmer, reprochant au législateur d'avoir nourri la confusion dans une matière déjà bien obscure ${ }^{19}$. Pourtant, une différence assez nette semble séparer ce nom commercial de la dénomination sociale : celle-ci, tout comme la raison sociale dans une société de personnes, constitue la véritable expression de l'identité d'une personne morale, son «état civil », tandis que l'on pourrait comparer le nom commercial, distinct de la raison sociale, à un pseudonyme ${ }^{20}$. D'ailleurs, l'article qui permet d'utiliser un nom commercial oblige la société à indiquer sa raison sociale dans les actes et documents destinés aux tiers, c'est-à-dire à se manifester sous son identité véritable.

Les sociétés commerciales en France, au Québec et en Ontario*

\begin{tabular}{|c|c|c|c|}
\hline France & \multicolumn{2}{|c|}{ Québec } & Ontario \\
\hline \multirow[b]{2}{*}{$\begin{array}{l}\text { Sociétés de personnes } \\
\text { - en nom collectif } \\
\text { - en commandite } \\
\text { simple }\end{array}$} & texte français & version anglaise & \multirow[b]{2}{*}{$\begin{array}{l}\text { - partnership } \\
\text { - limited partnership }\end{array}$} \\
\hline & $\begin{array}{l}\text { - en nom collectif } \\
\text { - en commandite }\end{array}$ & $\begin{array}{l}\text { - general partnership } \\
\text { - limited partnership }\end{array}$ & \\
\hline $\begin{array}{l}\text { Sociétés de capitaux } \\
\text { - anonyme } \\
\text { - en commandite } \\
\text { par actions }\end{array}$ & $\begin{array}{l}\text { compagnie } \\
\text { (soc. par actions) }\end{array}$ & \multirow[t]{2}{*}{$\begin{array}{l}\text { - company } \\
\text { (joint-stock } \\
\text { company) }\end{array}$} & \multirow[t]{2}{*}{$\begin{array}{l}\text { - business } \\
\text { corporation } \\
\text { (autrefois } \\
\text { company) }\end{array}$} \\
\hline $\begin{array}{l}\text { Type intermédiaire } \\
\text { - à responsabilité } \\
\text { limitée }\end{array}$ & & & \\
\hline
\end{tabular}

* Une case vide indique que le droit de l'Etat intéressé ne connaît pas de forme correspondante de société. Les correspondances établies n'impliquent évidemment pas une identité absolue des régimes. Il convient de signaler une différence importante entre les systèmes de droit français et ceux de common law : les premiers reconnaissent la personnalité morale même aux sociétés de personnes, tandis que les seconds ne la reconnaissent qu'aux sociétés de capitaux. Sur ce point, certains pays de droit civil (par exemple, l'Allemagne, la Suisse et l'Italie) adoptent une position analogue à celle du droit anglais.

\section{Le nom des sociétés de personnes en droit québécois}

A première vue, le Code civil semble suivre d'assez près le Code de commerce de France. En effet, l'article 1865 du Code civil dispose : «Les

18. Cette justification semble avoir été méconnue par certains auteurs ; voir Hémard, Terré et Mabilat, p. 232, no 248 : \& ce qui peut paraître une évidence ».

19. Par exemple, Bastian et Nocquet emploient carrément « dénomination sociale » au lieu de nom commercial " (op. cit., nos 11 et 22).

20. Voir, dans ce sens, Hémard, Terré et Mabilat, op. cit., p. 232, no 248 : \& Il convient donc de le [sc. le nom commercial] distinguer nettement de a la dénomination sociale » qui est le nom social des sociétés à responsabilité limitée et des sociétés par actions. \# Bastian et Nocquet, s'ils assimilent le nom commercial à une dénomination sociale, reconnaissent cependant cette analogie entre la dénomination sociale et la raison sociale (op. cit., nos 1,162 et 163). 
sociétés en nom collectif sont celles qui sont formées sous un nom collectif ou raison sociale, consistant ordinairement dans le nom des associés ou de l'un ou plusieurs d'entre eux... $\AA$ y regarder de plus près, on découvre cependant une particularité terminologique : alors que le Code de commerce, à l'article 21, portait que «les noms des associés peuvent seuls faire partie de la raison sociale », notre Code civil dit seulement que la raison sociale se compose « ordinairement» du nom des associés, ce qui laisse place à une « raison sociale » qui comprendrait autre chose que le nom des associés, c'est-à-dire une désignation que le droit français de l'époque appelait plutôt dénomination sociale ou raison commerciale.

Derrière ce qui peut paraître une subtilité terminologique, se cache une modification importante des règles régissant la désignation des sociétés de personnes. En droit français, cette société est tenue de se désigner par une raison sociale, formée du seul nom des associés indéfiniment responsables du passif social. Au contraire, le droit anglais laisse au partnership une liberté beaucoup plus grande dans le choix de son nom :

... partners may trade under any name they please, according to our law, whether it be a combination of their own several names or the names of others, or a name descriptive merely of their business, so long as they do not thereby fraudulently imply that their business is identical with some other competing one ${ }^{21}$.

Les codificateurs, en préférant la solution du droit anglais à celle du droit français, auraient pu respecter le sens habituel de « raison sociale ». En effet, il eût suffi de déplacer l'adverbe «ordinairement », pour obtenir : «... celles qui sont ordinairement formées sous... (une) raison sociale, consistant dans le nom des associés... Pourtant, cette formulation n'aurait guère été satisfaisante, puisqu'elle n'aurait pas établi de terme désignant de façon générique le nom de la société, sous les différentes formes qu'il pouvait prendre ${ }^{22}$.

La solution idéale aurait probablement été de laisser la société de personnes libre de choisir une raison sociale ou une dénomination sociale, un peu commes le législateur français l'a fait pour la société à responsabilité limitée. Cependant on ne peut guère reprocher aux codificateurs de ne pas avoir adopté cette solution. La traduction de firm name par raison sociale s'imposait avec une certaine évidence, ces deux termes désignant, chacun dans leur langue, l'appella-

21. Sir A. Underhill, Principles of the Law of Partnership, London, Butterworths, 1966, p. 41. On trouve la même règle énoncée en 1860 dans $N$. Lindley, $A$ Treatise on the Law of Partnership, Philadelphia, T. \& W. Johnson, p. 166 : * There is apparently nothing at common law to prevent persons from carrying on business under any name they please... \$ Le partnership ne possédant pas de personnalité distincte de celle des associés, il n'est qu'un groupe de personnes exerçant ensemble une activité lucrative sous un nom quelconque. Le droit québécois, à la suite du droit anglais, permet même à une personne qui exploite seule un commerce de faire suivre son nom de la mention < et compagnie \$ (Loi des déclarations des compagnies et sociétés, S.R.Q. 1964, chap. 272 , art. 10); le droit français et le droit suisse interdisent l'emploi d'un nom qui pourrait faire présumer l'existence d'une société, alors qu'elle n'existe pas.

22. On trouve une lacune analogue dans les textes français d'aujourd'hui, mais à propos d'un terme spécifique. En effet, le législateur n'a pas établi de terme désignant spécifiquement le $\$$ nom commercial, distinct de la raison sociale ». Peut-être l'usage finirat-il par transformer en terme spécifique nom commercial s, qui, dans l'état actuel, est un terme générique. 
tion des sociétés de personnes ${ }^{23}$. Ainsi, le Code civil a emprunté le terme « raison sociale » au droit français, mais lui a donné le sens du terme anglais firm name, d'ailleurs employé dans la version anglaise.

L'influence anglaise se reconnaît encore à la prédilection de nos textes anciens pour les doublets ou même les triplets, lorsqu'il s'agit de désigner la « raison sociale ». Dans un ouvrage de 1860 , un auteur anglais écrit :

A firm is usually described in legal proceedings as certain persons trading or carrying on business under, and using the name, style and firm of, \&c. ${ }^{24}$

Nos textes ont traduit fidèlement les modèles anglais, ce qui nous vaut des expressions du genre : «nom, titre ou raison ${ }^{25} »$ ou «nom et raison ${ }^{26} »$.

\section{Le nom commercial des personnes physiques en droit québécois}

Le terme anglais firm peut désigner une société de personnes ${ }^{27}$, mais aussi un établissement commercial, une maison, sans égard au fait que cet établissement soit exploité en société ou non. Dans les définitions, le droit anglais recourt souvent à l'énumération et, comme celle-ci est interprétée restrictivement, on préfère généralement en donner plus que moins, d'où des définitions du genre : "The word a person» includes a firm, partnership, corporation, club, association and syndicate ${ }^{28}$. $\gg$ Par suite de l'ambiguïté du mot firm, qui peut s'employer comme synonyme de firm name, le législateur en arrive à reconnaître la personnalité non seulement à la société, mais aussi à la raison sociale, traduisant la définition citée par : « Le mot «personne» inclut une raison sociale... $\gg$ et fixant plus loin les sanctions applicables aux infractions commises par la raison sociale.

En outre, comme le commerçant peut exploiter un établissement sous un nom également appelé en anglais firm name ou firm, on en arrive à parler de la raison sociale de personnes physiques ${ }^{29}$. Ainsi, c'est l'application hors de propos de l'équivalence établie entre firm name et raison sociale qui explique qu'au Québec on en soit venu à parler de raison sociale même en l'absence d'une

23. Raison sociale et firm name sont d'ailleurs partout donnés comme équivalents. Voir L. Goirand, The French Code of Commerce..., London, Stevens \& Sons, 1880, art. 20 et 21 ; Sir A. Underhill, op. cit., p. 41 ; de même les dictionnaires bilingues. Il convient de remarquer que l'anglais ne possède pas de terme courant qui corresponde exactement à raison sociale ; toutefois, le droit écossais semble avoir un tel équivalent strict : proper ou personal firm (Oxford English Dictionary, s.v. firm).

24. N. Lindley, op. cit., p. 180.

25. Loi des déclarations des compagnies et sociétés, déjà citée, art. 9, par. 1 et 4, et art. 13 .

26. Code civil, art. $1837,1870,1875$ et 1880 ; Loi des sociétés nationales de bienfaisance, S.R.Q. 1964, chap. 299, art. 2.

27. Par exemple, la loi anglaise intitulée The Partnership Act, 1890, art. 4 : e Persons who have entered into partnership with one another are for the purposes of this Act called collectively a firm.

28. Loi de la manutention de la gazoline, S.R.Q. 1964, chap. 75, art. 2 ; cette loi a été remplacée par L.Q. 1972, chap. 30. On trouvera d'autres énumérations du genre dans les textes suivants, où le mot firm est rendu par raison sociale: S.R.Q. 1964, chap. 46, art. 1er, chap. 52 , art. 2 , chap. 74 , art. 2 (loi remplacée par L.Q. 1972, chap. 30 ).

29. Voir les dispositions qui se trouvent assez curieusement données dans le Code civil au titre «De la société \$ (art. 1934b : une personne faisant affaires seule sous une raison sociale ») et dans la Loi des déclarations des compagnies et sociétés, S.R.Q. 1964, chap. 272, art. 10 et 19. 
société. Placé devant un problème de traduction analogue, le droit suisse a reculé devant une extension aussi audacieuse du sens de raison sociale. En effet, l'allemand, langue originale du Code des obligations, englobe sous un seul terme, Firma, les réalités que désignent, en droit français, les termes raison sociale, dénomination sociale, raison commerciale et nom commercial. Cependant, après avoir rendu uniformément Firma par raison sociale dans les dispositions relatives aux sociétés, la version française passe à « raison de commerce » dans le titre qui traite du nom commercial des personnes physiques et des sociétés ${ }^{30}$. Une solution analogue aurait été possible au Québec et elle aurait peut-être attiré l'attention sur ce qu'il y avait d'insolite à traiter des personnes physiques dans des textes visant les sociétés.

\section{Le nom des sociétés de capitaux en droit québécois}

Le Code civil ne consacre que trois articles (1889-1891) aux sociétés de capitaux ; en effet, le régime de ces sociétés est défini dans la Loi des compagnies (S.R.Q. 1964, chap. 271), d'inspiration anglaise, plutôt que dans le Code civil au titre \& Des sociétés », d'inspiration française. L'article 1890 du Code civil, suivant d'assez près les articles 29 et 30 du Code de commerce français, dispose : "Les noms des associés ou actionnaires ne paraissent pas dans les sociétés par actions qui sont généralement connues sous une dénomination qui indique l'objet de leur formation. Ce texte se conforme donc à la terminologie du droit français en appelant dénomination le nom de la société par actions. Cependant, le terme a dénomination », employé dans ce texte relégué dans l'oubli par la Loi des compagnies, n'était pas destiné à connaître une brillante carrière au Québec. Exception faite de quelques articles de la Loi des compagnies ${ }^{31}$, où l'emploi de dénomination fait plutôt l'impression d'une coquetterie de traducteur à la recherche d'un style raffiné, il faudra attendre les années 1970 pour voir le terme faire une réapparition timide dans les textes québécois ${ }^{32}$. Par contre, dans un projet de loi récent ${ }^{33}$, le gouvernement fédéral propose de traduire corporate name par dénomination sociale, s'alignant ainsi sur la terminologie française.

En anglais, le nom de la company peut être désigné par deux termes : company name et corporate name. Comme aucun de ces termes n'évoque

30. Code des obligations, Titre trente et unième : Des raisons de commerce.

31. Loi des compagnies, S.R.Q. 1964, chap. 271, art. 7(1), 215a et formules 2 et 16 en annexe. Dans les trois cas, le texte anglais est sensiblement le même et \& dénomination sociale " correspond a therewith (c'est-a-dire with the corporate name of any other company); or, dans la phrase même, corporate name a déjà été rendu par \& nom de compagnie $\gg$.

32. A notre connaissance, le terme refait surface aux endroits suivants : Loi sur la langue officielle (abrogée), L.Q: 1974, chap. 6, art. 11 (organismes gouvernementaux) ; Loi constituant la Société québécoise d'information juridique, L.Q. 1975, chap. 12, art. 1er; Charte de la langue française, L.Q. 1977, chap. 5, art. 14 (gouvernement, ministres, organismes de l'Administration et leurs services) et art. 34 (ordres professionnels). Ces textes appellent deux remarques. D'abord, deux de ces lois, portant sur la langue, témoignent d'un souci évident de correction. Ensuite, aucun de ces textes ne se rapporte à des sociétés, puisque SOQUIJ, malgré sa dénomination, est simplement un organisme de l'Administration, doté de la personnalité morale.

33. Bill S-2 de 1978, Loi modifiant la Loi sur les corporátions commerciales canadiennes, art. 6(1) de l'annexe; la distinction à faire entre raison sociale et dénomination sociale avait été soulignée dans C. Fortin et.N. Sénécal, Un nom d'emprunt pour les compagnies faisant affaires au Québec : pourquoi pas ? $\$,(1972) 32 \cdot R$. du $B, 473$, note 2 . 
directement la dénomination de l'article 1890 du Code civil, on les traduira généralement par nom de la compagnie ou nom ${ }^{34}$. Quant au terme *nom corporatif », les textes québécois, bien qu'ils l'emploient à l'occasion, ne semblent pas l'avoir adopté comme terme technique ${ }^{35}$. Il en va de même dans les textes fédéraux, y compris dans la Loi sur les corporations commerciales canadiennes, qui n'emploie qu'une fois le terme «nom corporatif ${ }^{36}$ ». Ces traductions transparentes n'ont pas toujours trouvé grâce aux yeux des traducteurs consciencieux qu'étaient nos rédacteurs de lois; c'est ainsi qu'on a parfois appliqué aux sociétés par actions deux synonymes de raison sociale, peu employés en droit français : nom social et nom collectif ${ }^{37}$. Quant au terme « raison sociale », on ne le trouve au sens de corporate name que depuis les années 1970, et dans un bien petit nombre de textes ${ }^{38}$.

\section{Raison sociale comme terme générique en droit québécois}

Malgré quelques hésitations, on peut néanmoins affirmer que le droit québécois, jusqu'au début de la décennie en cours, avait préservé dans sa terminologie une opposition entre la raison sociale de la société de personnes et le nom de la compagnie ${ }^{39}$. Cependant, cette opposition, qui s'était substituée à l'opposition du droit français entre raison sociale et dénomination sociale, avait été infléchie dans le sens de l'opposition anglaise entre firm name et corporate name. Dès la rédaction du Code civil, on avait abandonné la règle de composition de la raison sociale, en admettant qu'elle puisse comprendre autre chose que le nom d'associés; puis on avait laissé de côté la règle d'emploi, en appelant

34. Par exemple, Loi des compagnies, déjà citée, art. $7,8,19,20$ et 21 . Le Code civil, dans le titre \& Des corporations \$, qui, il est vrai, ne se rapporte qu'indirectement aux compagnies, rend corporate name par nom propre $\$$

35. Dans la Loi des compagnies, on ne le trouve que dans les formules 2 et 16 en annexe. Parmi les innombrables lois définissant le régime particulier de certains types de sociétés, il semble que deux seulement emploient ce terme : Loi des compagnies de télégraphe et de téléphone, S.R.Q. 1964, chap. 286, art. 2 et Loi des sociétés de prêts et de placements, S.R.Q. 1964 , chap. 289 , art. 5.

36. S.C. 1975, chap. 33, art. 10(6) ; dans l'ancienne Loi sur les corporations canadiennes, S.R.C. 1970, chap. C-32, on trouvait \& nom corporatif $\$$ aux articles $7(2) \mathrm{b}, 9(4)$ et 159(2). Dans le projet de loi S-2, déjà cité, qui contient une nouvelle version française de la loi de 1975, corporate name est rendu par dénomination sociale.

37. Nom social : Loi des sociétés d'exploration minière, S.R.Q, 1964, chap. 284, art. 5 et Loi des assurances (abrogée), S.R.Q. 1964, chap. 275, art. 3. Il a déjà été question du sens de ce terme en droit français à la note 4 ci-dessus. Nom collectif : Loi des compagnies de gaz, d'eau et d'électricité, S.R.Q. 1964, chap. 285, art. 5 ; ce terme apparaît cependant, aussi bien en droit québécois qu'en droit français, dans le nom d'un type de société : la société en nom collectif. A propos de la synonymie avec raison sociale, voir Hémard, Terré et Mabilat, op. cit., no 245, p. 225. D'ailleurs, on trouve à l'article 1865 du Code civil du Québec : \& sous un nom collectif ou raison sociale 》.

38. Abstraction faite des lois en matière linguistique, qui emploient ce terme en un sens encore plus large, on trouve raison sociale au sens de corporate name dans la Lot sur les assurances, L.Q. 1974, chap. 70, art. 24, 106, 108, 186 et 194, ainsi que dans la Loi constituant la Société du parc industriel et commercial aéroportuaire de Mirabel, L.Q. 1976, chap. 32, art. 2. Vraisemblablement, cette extension a été favorisée par l'emploi de plus en plus répandu de société dans des cas où l'usage antérieur disait compagnie ou corporation. Il convient de remarquer que le droit suisse, également assujetti aux vicissitudes de la traduction, emploie raison sociale pour désigner le nom des sociétés de capitaux (p. ex., Code des obligations, art. 620). On trouve le même emploi dans la Loi sur les corporations, Lois révisées du Nouveau-Brunswick, 1973, chap. C-13, art. 6(1) et $11(2)$. 39. Par exemple, la Loi des déclarations des compagnies et sociétés, déjà citée, respecte très 
raison sociale la raison de commerce des personnes physiques. D'autre part, le droit québécois reconnaissant la personnalité morale aux sociétés de personnes, l'opposition entre raison sociale et nom de la compagnie ne pouvait se réclamer de la même justification que l'opposition correspondante entre firm name et corporate name ${ }^{40}$. Ainsi tiraillé entre deux systèmes juridiques, sans épouser les règles ni de l'un ni de l'autre, le terme « raison sociale » était devenu un terme fou, au sens où l'on dit un moteur fou, une aiguille folle.

En effet, dès le moment où, dans le cas des compagnies, on rend corporate name par raison sociale, terme qui correspondait jusque-là à firm name, on supprime dans la terminologie du droit québécois toute trace de l'opposition entre firm name et corporate name; or nous avons vu que la règle d'emploi de « raison sociale \& dépendait, en droit québécois, de cette opposition. Après avoir élargi le sens du terme « raison sociale » pour en faire l'équivalent de firm name, on finit par lui donner aussi le sens de corporate name. Cependant, le terme corporation ne désigne pas seulement des sociétés, c'est-à-dire des groupements à but lucratif : il s'étend à toute entité dotée de la personnalité morale, qu'elle soit formée ou non en vue d'un but lucratif et qu'elle relève du droit privé ou du droit public. Il ne faut donc pas s'étonner du fait que la Charte de la langue française, après avoir établi qu'une raison sociale en français est nécessaire à l'obtention de la personnalité juridique (art. 65), parle de la raison sociale des associations à but non lucratif et même des services de santé ou des services sociaux. Cette nouvelle extension de sens accroît encore la distance qui sépare l'usage québécois de l'usage français, où raison sociale ne s'emploie pas à propos de personnes morales autres que des sociétés ${ }^{41}$.

Si le terme « raison sociale » possède un sens très précis en droit français, on ne peut dire qu'il en soit ainsi en droit québécois. En effet, par suite des vicissitudes de son histoire, ce terme s'est trouvé à peu près dépourvu de toute signification propre, si ce n'est celle d'un vague synonyme de nom. On serait bien en peine de lui faire correspondre un terme quelconque tiré du droit français ou du droit anglais ${ }^{42}$. Même un terme aussi général que «nom des personnes morales » ne conviendrait pas, puisqu'on parle également de la raison sociale des personnes physiques. Nom commercial doit aussi être rejeté, dans la mesure où raison sociale s'emploie à propos de groupements à but non lucratif.

40. Voir Halsbury, cité dans Words and Phrases Legally Defined, ed. by J.B. Saunders, London, Butterworths, 1969 , s.v. firm : * The firm name is not the name of a corporation; it is a short name for $X, Y$ and $Z$, carrying on business in partnership. $D$ De même, dans le cas du commerçant, le firm name n'est qu'un nom de convention, servant à désigner le commerçant, qui n'est pas une corporation, mais une personne physique.

41. Selon l'article 5 de la Loi du $1^{\text {er }}$ juillet 1901 relative au contrat d'association, l'association est désignée par un titre, terme auquel on substitue parfois dénomination. Le texte correspondant de Belgique Loi du 27 juin 1921 accordant la personnalité civile aux associations sans but lucratif et aux établissements d'utilité publique, emploie tantôt dénomination, tantốt dénomination sociale. Dans le cas des personnes morales de droit public, les droits français et belge emploient dénomination ou nom.

42. Ainsi qu'en témoignent les difficultés éprouvées par les traducteurs chargés de la version anglaise de nos lois. Ils ont su éviter le piège lorsque raison sociale servait à désigner le nom d'une corporation (voir la version anglaise des deux lois citées à la note 38), mais dans les textes où le terme reçoit toute son extension, ils se sont contentés de la rendre par firm name, ce qui aurait pour effet de dispenser les corporations de franciser leur nom (voir la Charte de la langue française, art. 63 et antérieurement la Lot sur la langue officielle, L.Q. 1974, chap. 5, art. 30). 


\section{Conclusion}

S'il est souhaitable de rétablir une saine terminologie dans notre langue juridique, il faut aussi éviter de porter atteinte à l'autonomie de notre droit. Sur le fondement de ce principe, voici les modifications qu'il faudrait apporter à notre terminologie :

1) Dans le cas des sociétés de personnes, on pourrait convenir qu'elles peuvent se désigner ou par une RAISON SOCIALE (composée exclusivement du nom d'associés indéfiniment responsables) ou par une DENOMINATION SOCIALE (de composition libre).

2) Dans le cas de personnes physiques exerçant une activité commerciale, il faudrait parler de NOM COMMRRCIAL, puisque « raison de commerce \& a vieilli.

3) Pour les sociétés de capitaux (nos * compagnies à fonds social »), il y aurait lieu d'adopter DÉNOMINATION SOCIALE.

4) En ce qui concerne les associations (nos * corporations sans capitalactions $\gg$ ), on serait justifié de préférer DÉNOMINATION (SOCIALE) au terme c titre ১, employé par la loi française, d'autant que notre droit les regroupe avec les sociétés de capitaux sous le terme générique de corporation.

5). Les personnes morales de droit public seraient désignées par une DÉNOMINATION, qui ne deviendrait DÉNOMINATION sociale que dans les cas où elles auraient la forme d'une société.

6) Comme terme générique, on pourrait employer NOM COMMERcial quỉ engloberait les cas 1,2 et 3 ou l'expression DENOMINATION ou RAISON SOCLALE qui couvrirait les cas 1,3 et 4 .

Selon une formule souvent citée, le droit québécois constitue un « modèle vivant de droit comparé ». Sans mettre en doute la vérité de la formule, le linguiste ne peut échapper à l'impression qu'elle ne paraît possible que du point de vue de Sirius. En effet, l'étude approfondie de notre langue juridique le conduirait plutôt à croire que le droit québécois est un laboratoire vivant où se manifestent toutes les formes de la contamination linguistique. La présente recherche, à propos d'un cas sans doute exemplaire, a voulu montrer comment la cohabitation de deux systèmes de droit sur un même territoire peut entraîner, par des interférences en chaîne, une altération profonde de la terminologie juridique. 